\title{
INFLUENCE OF SELECTED EXAMINATION POSTURES ON SHAPE OF THE SPINE AND POSTURAL STABILITY IN HUMANS
}

\author{
Jakub Krejci ${ }^{\mathrm{a}}$, Jiri Salinger ${ }^{\mathrm{a}}$, Jiri Gallo ${ }^{\mathrm{b}}$, Petr Kolisko ${ }^{\mathrm{c}}$, Petr Stepanik ${ }^{\mathrm{a}}$ \\ ${ }^{a}$ Department of Biomechanics and Engineering Cybernetics, Faculty of Physical Culture, Palacky University, Olomouc, \\ Czech Republic \\ ${ }^{b}$ Department of Orthopaedics, Faculty of Medicine and Dentistry, Palacky University, Olomouc, Czech Republic \\ c Department of Functional Anthropology and Physiology, Faculty of Physical Culture, Palacky University, Olomouc, Czech \\ Republic \\ e-mail: jiri.gallo@volny.cz
}

Received: October 22, 2008; Accepted (with revision): November 24, 2008

Key words: Spinal deformity/Measurement/Non-radiographic/Diagnostic system/Examination postures/Postural stability/ Approximation polynomial

Aims: The accuracy of non-radiographic measurement of the spinal shape is influenced by postural sway (PS). The aim of this study was to determine whether certain examination postures prevent PS without changing key spinal characteristics.

Methods: We tested 1) natural standing position (posture A), 2) fixation postures standing with the support of the upper limbs against a wall (posture B) and 3) standing with the support of the head and chest against a wall (posture C). There were examined 60 subjects; the examination of each posture was repeated five times in each individual by DTP-3 microcomputer position system. The spinal shape changes resulting from tested postures were assessed using $t$-test. The role of PS was assessed using standard deviations, and the significance of differences between the individual postures was determined using the $F$-test.

Results: Compared to posture A, the fixation posture B did not have any significant influence on the degree of PS, but it significantly influenced the shift of the spine from the ideal vertical and its slope, although this did not result in spine curve deformation. The fixation posture $\mathrm{C}$ significantly reduced the PS compared to posture A; however, there were significant changes in the spinal shape. This fixation posture shifted significantly the spine from the ideal vertical and slope characteristics.

Conclusion: PS plays a role in the examination of spinal shape. Positions B and C did not meet the required criteria. Therefore, it is necessary to search for another examination position.

\section{INTRODUCTION}

To reduce cumulative radiation exposure, especially in children and adolescents, non-radiographic methods for spinal shape examination have been introduced ${ }^{1-6}$. However, random variations in the standing position (postural sways) of the examinee caused by postural instability or breathing can influence the accuracy and reliability of the methods developed for spinal shape measurement. Moreover, patients with scoliosis have much less control over postural stability than the normal population ${ }^{7,8}$. Taken together, there is a need for examination postures that may minimize the influence of postural sway on the accuracy of measurement.

From the physical point of view, postural sways may be described by the amplitude and period of spatial body deflections, and therefore a single examination of spinal shape shows only the momentary body posture during a series of deflections. One method for resolving this problem is to repeat spinal shape examinations within an appropriate time interval with the subjects in different examination postures. However, any fixation procedure that reduces the amplitude of postural sway may change the body posture and the spinal shape simultaneously. Therefore, it is important to determine the characteristics of selected examination postures in terms of both the postural stability and the influence of body posture on spinal shape. An accurate description of these characteristics of examination postures will enable standardization of such conditions as well as a comparison of results obtained with different methods.

In this study the natural standing position was compared with two postures that utilize a different degree of fixation. The aim was to evaluate the influence of each position on the degree of postural sway and on spinal shape. Ideally, the best position is that with minimal postural sway while maintaining the spinal shape as close as possible to that of the natural standing position.

\section{MATERIALS AND METHODS}

\section{Investigated examination postures}

The examination postures (Fig. 1) tested in the study were: 


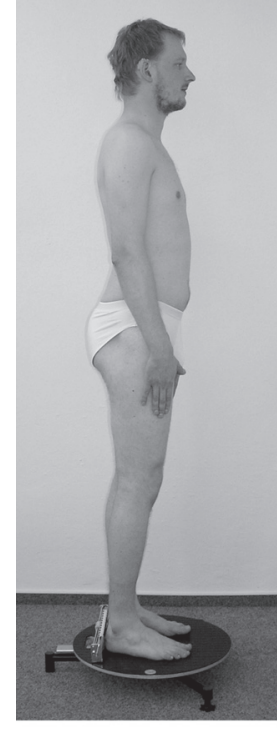

Posture A

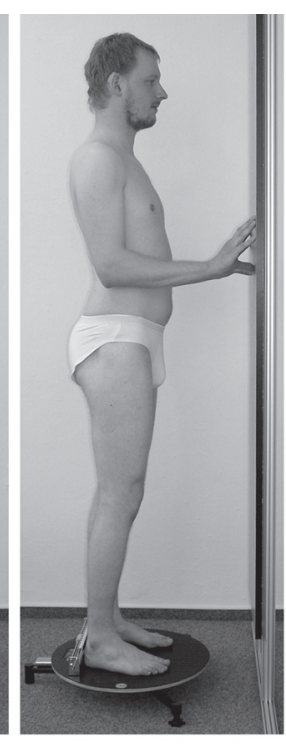

Posture B

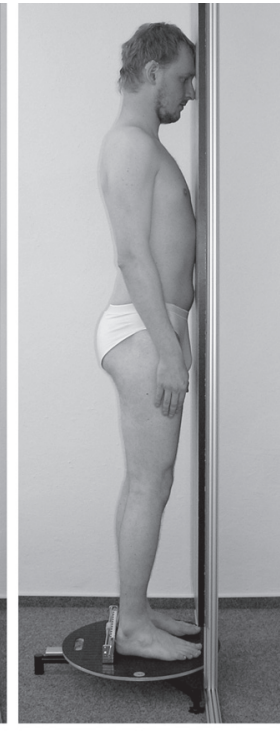

Posture C
Fig. 1. Natural standing position (posture A) and proposed fixation postures $\mathrm{B}$ and $\mathrm{C}$

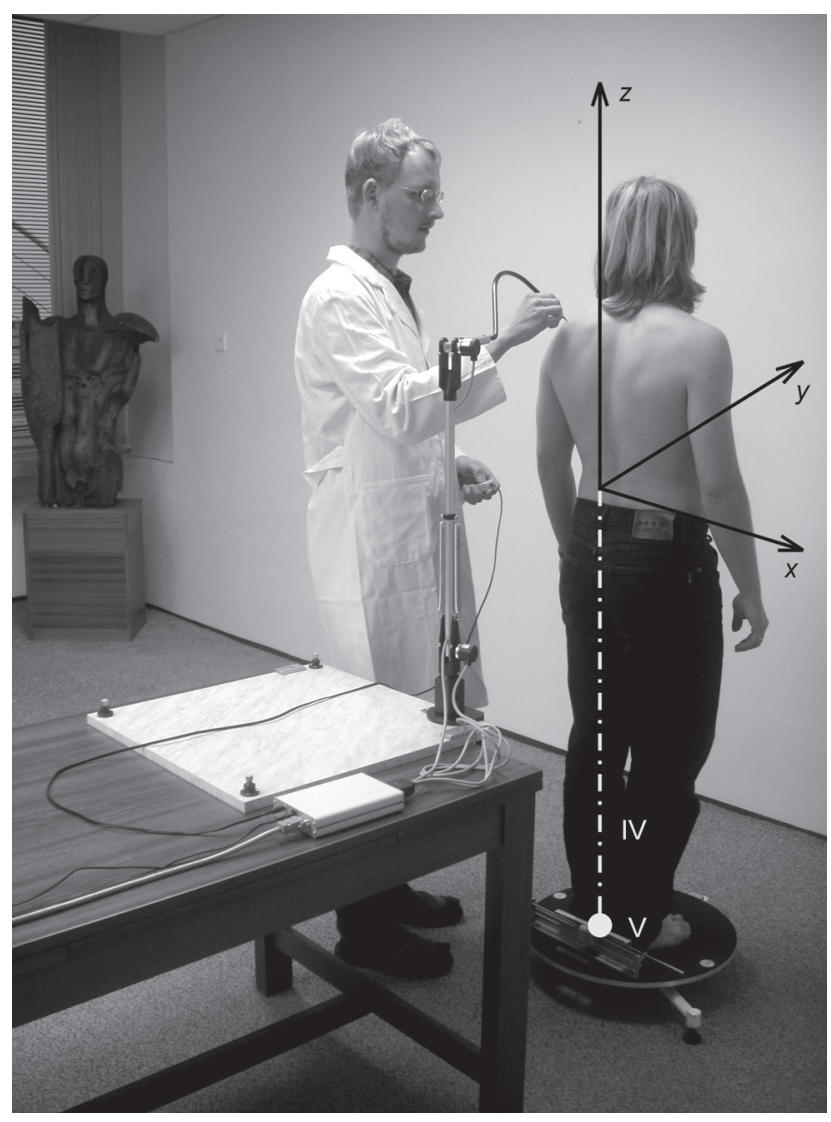

Legend

$x, y, z$ - coordinate axes

$\mathrm{V}-$ point for erection of ideal vertical IV - ideal vertical

Fig. 2. Examination of spinal shape and postural sway using the DTP-3 diagnostic system
- Posture $A$ is the natural standing position without fixation. The width of the position is habitual, with the upper limbs alongside the body; the examinee is looking straight ahead.

- Fixation posture $B$ is a posture in which the upper limbs are supported against a vertical wall. The width of the position is habitual, the forearms are horizontal, and the examinee touches the wall with their fingertips and looks straight ahead.

- Fixation posture $C$ is a posture in which the head and chest of examinee are supported against a vertical wall. The width of the position is habitual, the upper limbs lie alongside the body, and the forehead, tip of the nose, and the chest are touching the wall.

\section{The instrument}

We used the microcomputer diagnostic system, DTP-3, which allowed non-invasive examination of spinal shape in the sagittal and frontal planes by applying the contact method $^{9}$. The DTP-3 software involved algorithms for calculating postural sway amplitudes and for determining spinal curvature by calculating the coefficients of approximation polynomials ${ }^{3,9}$. This system allowed repeated examinations within a short time interval. The coordinates of anatomic points on the skin surface of the examinee were registered with a position sensor and transferred to a personal computer, which converted these points into output records for subsequent evaluation. The postural sensor allowed measurement of the positions of points in the sphere with a diameter of $2200 \mathrm{~mm}$ and a standard error of $0.5 \mathrm{~mm}^{9-11}$.

When using the DTP-3 system to diagnose spinal shape (Fig. 2), we palpated and detected the skin projections of the following anatomic points: left and right lateral parts of the acromion, bilateral posterior superior iliac spine, and the processus spinosus of vertebrae $\mathrm{C} 3-\mathrm{C} 7, \mathrm{~T} 1-\mathrm{T} 12$, and L1-L5. The positions of the measured points were converted into output records. The so-called ideal vertical (IV), i.e. mathematical simulation of a plumb line erected from the centre of the intercalcaneal line $(\mathrm{V})$, was used to evaluate the spinal shape. The orientation of the 3D Cartesian coordinate system was as follows: axis $z$ was on the ideal vertical and oriented in the caudal-cranial direction, axis $x$ was parallel with the intercalcaneal line and in left-right direction, and the direction of axis $y$ was in the posterior-anterior direction. As a result, frontal plane was defined by axes $x z$ and the sagittal plane by axes $y z$.

\section{Study group}

The experimental part of the study included measurements of 60 subjects: 26 men and 34 women, aged $22.8 \pm 1.3$ years (mean $\pm \mathrm{SD}$ ), weighing $67.4 \pm 9.6 \mathrm{~kg}$, and $174.1 \pm 9.5 \mathrm{~cm}$ tall; the height of the spine given by the vertical distance between the processus spinosus of $\mathrm{C} 3$ and L5 was $52.0 \pm 3.9 \mathrm{~cm}$. The group included only healthy subjects. The examination of spinal shape in individuals was repeated five times for each of the three postures (A, $\mathrm{B}, \mathrm{C}$ ), and individual measurements followed immediately one after the other. The duration of one measurement was 


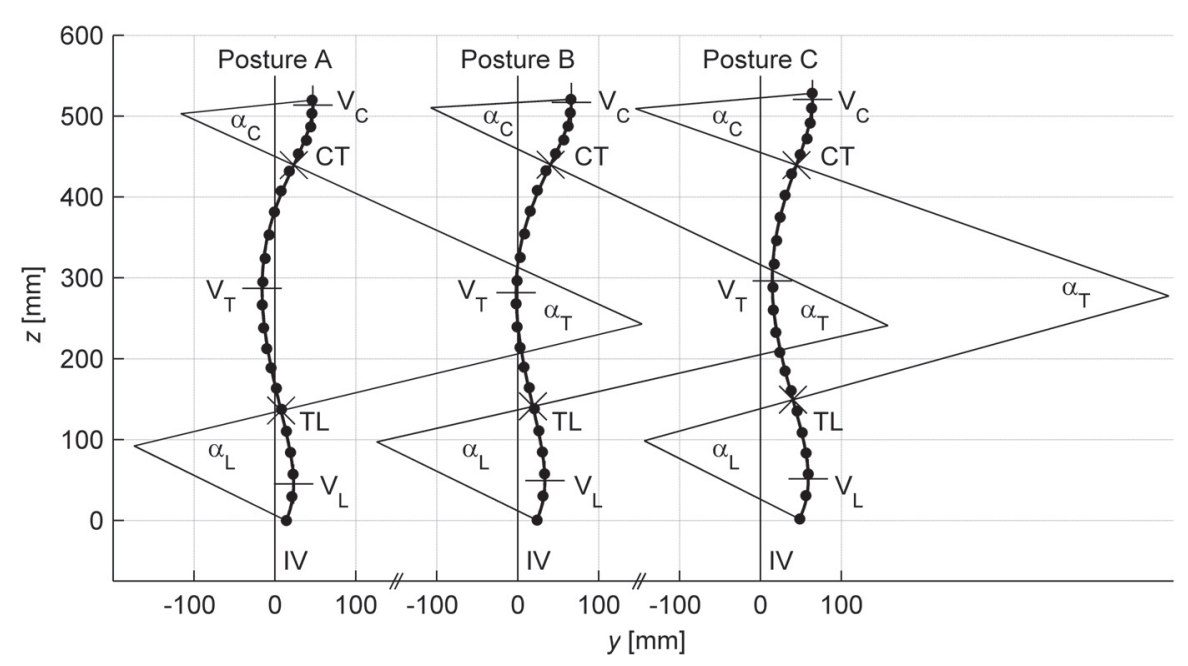

Legend

CT - cervicothoracic transition

TL - thoracolumbar transition

$\alpha_{C}$ - angle of cervical lordosis

$\alpha_{T}-$ angle of thoracic kyphosis

$\alpha_{L}$ - angle of lumbar lordosis

IV-ideal vertical

$V_{C}$ - cervical lordosis peak

$V_{T}$ - thoracic kyphosis peak

$V_{L}$ - lumbar lordosis peak

Fig. 3. Mean angle parameters and spinal shapes for postures A, B, and C
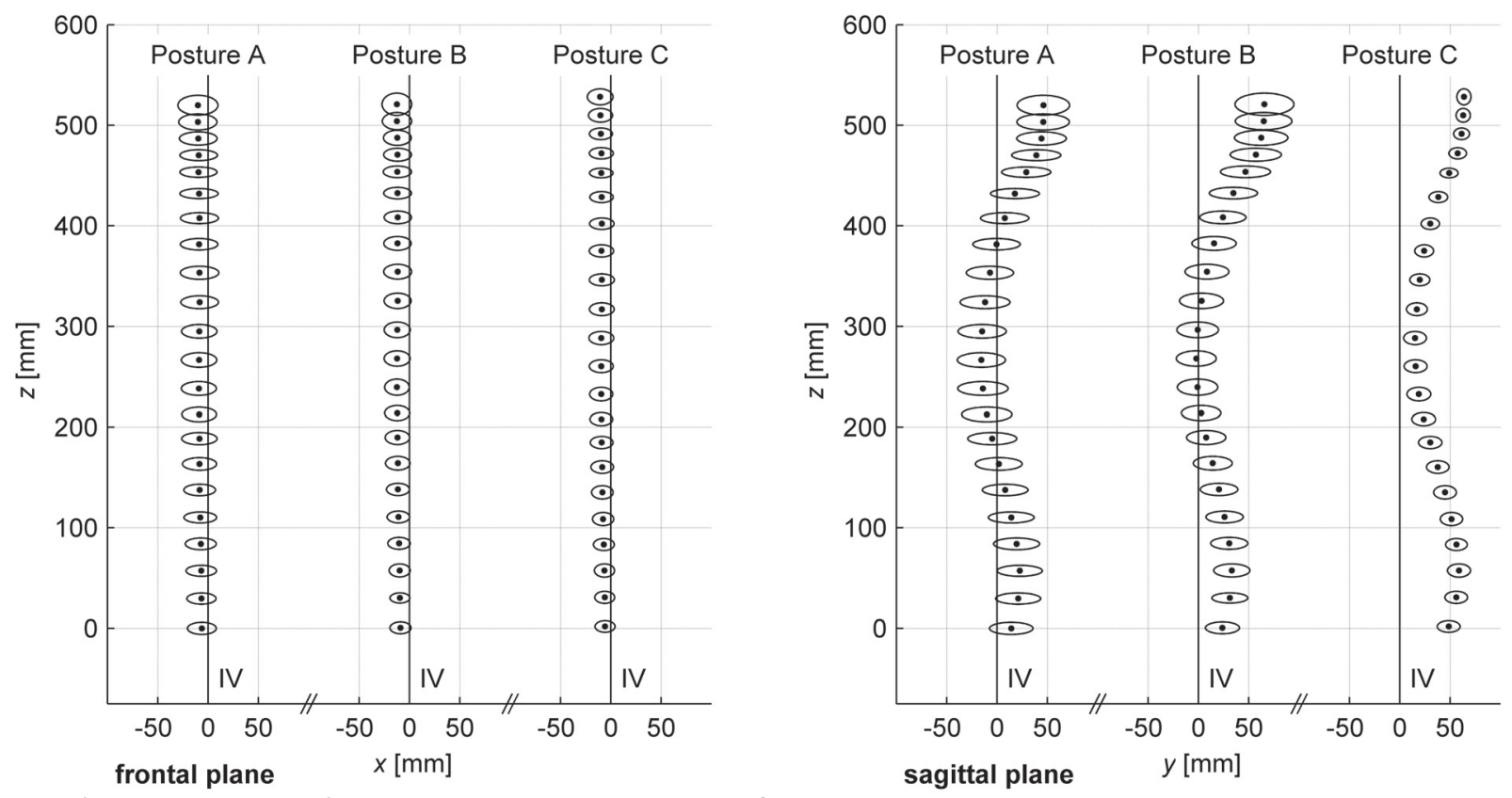

Legend

- mean position of processus spinosus

$\bigcirc$ - mean of standard deviations of processus spinosus (enlarged five times)

IV - ideal vertical

Fig. 4. Mean positions and mean of standard deviations of the processus spinosus in the 60 subjects in postures A, $\mathrm{B}$, and $\mathrm{C}$

$32.2 \pm 4.6$ s. Spine height was standardized so that the results of subjects of different height could be compared.

\section{Evaluation of the postural stability}

The stability of selected processus spinosus of individual subjects was evaluated by standard deviations (SDs) calculated from five repeated measurements of coordinates of the selected processus spinosus in all monitored postures $^{9,12}$. Based on these, mean of SDs (MSD) in coordinates $x, y$, and $z$ were calculated for the whole group.

\section{Evaluation of the spinal shape with approximation polynomial}

Spinal shape is given by the mean coordinates of all processus spinosus calculated from five repeated measurements of their coordinates. The repeated measurement and averaging improves the reliability of measurements as it reduces the influence of postural sway on the examinee.

Direct evaluation of 3D coordinates $x_{i}, y_{i}$, and $z_{i}$ of all processus spinosus, $i=1,2, \ldots, 22$, for all subjects is time- 
Table 1. Mean of standard deviations of processus spinosus coordinates $\mathrm{x}$ and $\mathrm{y}$ in the 60 subjects for postures $\mathrm{A}, \mathrm{B}$, and $\mathrm{C}$

\begin{tabular}{|c|c|c|c|c|c|c|c|c|c|c|c|c|c|c|c|}
\hline \multirow{4}{*}{$\begin{array}{l}\text { Processus } \\
\text { spinosus }\end{array}$} & \multicolumn{8}{|c|}{$x$ coordinate } & \multicolumn{7}{|c|}{$y$ coordinate } \\
\hline & \multicolumn{3}{|c|}{ Posture } & \multicolumn{5}{|c|}{ Comparison } & \multicolumn{3}{|c|}{ Posture } & \multicolumn{4}{|c|}{ Comparison } \\
\hline & \multirow{2}{*}{$\begin{array}{c}\text { A } \\
\text { MSD } \\
\end{array}$} & \multirow{2}{*}{$\begin{array}{c}\text { B } \\
\text { MSD }\end{array}$} & \multirow{2}{*}{$\begin{array}{c}\mathrm{C} \\
\mathrm{MSD} \\
\end{array}$} & \multirow{2}{*}{\multicolumn{3}{|c|}{$\begin{array}{c}\mathrm{B}-\mathrm{A} \\
\Delta\end{array}$}} & \multirow{2}{*}{\multicolumn{2}{|c|}{$\begin{array}{c}\mathrm{C}-\mathrm{A} \\
\Delta \\
\end{array}$}} & \multirow{2}{*}{$\begin{array}{c}\text { A } \\
\text { MSD }\end{array}$} & B & $\mathrm{C}$ & \multirow{2}{*}{\multicolumn{2}{|c|}{$\begin{array}{c}\mathrm{B}-\mathrm{A} \\
\Delta\end{array}$}} & \multicolumn{2}{|l|}{$C-A$} \\
\hline & & & & & & & & & & MSD & MSD & & & $\Delta$ & \\
\hline C3 $[\mathrm{mm}]$ & 3.99 & 2.94 & 2.55 & 1.05 & $\dagger$ & & $-1.45 \dagger$ & $\dagger$ & 5.22 & 5.85 & 1.40 & 0.63 & & $-3.82 \dagger$ & $\dagger$ \\
\hline $\mathrm{C} 4 \quad[\mathrm{~mm}]$ & 3.81 & 2.90 & 2. & -0.91 & & & $-1.41 \dagger$ & $\dagger *$ & $J$. & & 1. & & & $-3.81 \dagger$ & $\dagger$ \\
\hline C5 $[\mathrm{mm}]$ & 3.75 & & & & & & $-1.46 \dagger$ & $\dagger$ & 4.5 & & 1. & & & $-3.36 \dagger$ & $\dagger$ \\
\hline C6 $[\mathrm{mm}]$ & & & & & & & -1.34 & $\dagger$ & & & & & & $-3.14+$ & $\dagger$ \\
\hline C7 [mm] & 3. & & & & & & $t$ & $\dagger$ & & & & & & $10 \dagger$ & $\dagger$ \\
\hline $\mathrm{T} 1 \quad[\mathrm{~mm}]$ & 3. & & & -1.05 & $\dagger$ & & 9 & $\dagger$ & 4.8 & 4. & 1.8 & -0.07 & & $-3.01 \dagger$ & $\dagger$ \\
\hline $\mathrm{T} 2 \quad[\mathrm{~mm}]$ & & & & 06 & $\dagger$ & & -1.34 & $\dagger$ & $4 . c$ & & 1. & & & $-2.98 \dagger$ & $\dagger$ \\
\hline $\mathrm{T} 3 \quad[\mathrm{~mm}]$ & & & & 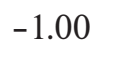 & $\dagger$ & & $t$ & $\dagger$ & & & & & & 1 & $\dagger$ \\
\hline $\mathrm{T} 4 \quad[\mathrm{~mm}]$ & 3.8 & & 2.50 & -1.06 & $\dagger$ & & -1.34 & $\dagger$ & 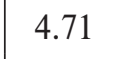 & & & 5 & & $-2.72 \dagger$ & $\dagger$ \\
\hline $\mathrm{T} 5 \quad[\mathrm{~mm}]$ & 3.76 & & 2.4 & -1.07 & $\dagger$ & * & -1.30 & $\dagger$ & 40 & & 2. & -0.59 & & $-2.89 \dagger$ & $\dagger$ \\
\hline $\mathrm{mm}]$ & & & & & & & 06 & $\dagger$ & & & & 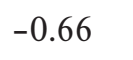 & 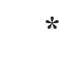 & $-2.54 \dagger$ & $\dagger$ \\
\hline $\mathrm{mm}]$ & & & & & & & 4 & $\dagger$ & & & & 0.02 & * & $-2.51 \dagger$ & $\dagger$ \\
\hline $\mathrm{T} 8 \quad[\mathrm{~mm}]$ & & & & -1.05 & $\dagger$ & & -1.20 & $\dagger *$ & & & & $-1.00 \dagger$ & $\dagger *$ & $-2.65 \dagger$ & $\dagger$ \\
\hline T9 [mm] & 3.45 & & & -0.95 & & & -1.19 & $\dagger *$ & 5 & & & $-1.08 \dagger$ & $\dagger *$ & $-2.64 \dagger$ & $\dagger$ \\
\hline $\mathrm{T} 10 \quad[\mathrm{~mm}]$ & 3.5 & & & -1.09 & $\dagger$ & & 26 & $\dagger$ & & & & & * & $-2.55 \dagger$ & 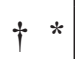 \\
\hline $\mathrm{T} 11$ [mm] & 3.40 & & & -0.95 & & & -1.15 & $\dagger$ & 4.6 & & & -0.78 & * & $-2.41 \dagger$ & $\dagger$ \\
\hline $\mathrm{T} 12$ [mm] & & & & -0.87 & & & -1.01 & $\dagger$ & & & & & * & $-2.29 \dagger$ & $\dagger$ \\
\hline $\mathrm{L} 1 \quad[\mathrm{~mm}]$ & 3.2 & & 2. & -1.01 & $\dagger$ & & -1.16 & $\dagger$ & & & 2. & & 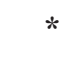 & $-2.33 \dagger$ & $\dagger$ \\
\hline $\mathrm{L} 2 \quad[\mathrm{~mm}]$ & 3.09 & & & & & & -1.01 & $\dagger$ & & & & -0.88 & 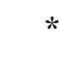 & $-2.42 \dagger$ & $\dagger$ \\
\hline $\mathrm{L} 3 \quad[\mathrm{~mm}]$ & 3.03 & & & & & & -1.03 & $\dagger$ & 4.51 & & 2. & -0.89 & 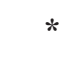 & $-2.21 \dagger$ & $\dagger$ \\
\hline $\mathrm{L} 4 \quad[\mathrm{~mm}]$ & 2.91 & 1.99 & & -0.92 & & & -0.92 & ; & 4.4 & 3.5 & 2.2 & -0.91 & 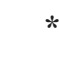 & $-2.22 \dagger$ & $\dagger$ \\
\hline L5 [mm] & 2.88 & & 2.00 & -0.77 & & & -0.88 & & 4.3 & 3.39 & 2. & -0.95 & A & $-2.06+$ & $\dagger$ \\
\hline
\end{tabular}

Legend

MSD - mean of standard deviations in the respective coordinate

$\Delta$ - parameter difference

$\dagger-|\Delta| \geq 1 \mathrm{~mm}$

* $-\mathrm{P}<0.05$ (F-test)

consuming. Therefore, a computer algorithm was used to evaluate the spinal shape, applying coefficients of the approximation polynomial $1^{9,13}$. The clinical interpretation of calculated polynomial coefficients $b_{0}, b_{1}, b_{2}, b_{3}, b_{4}, b_{5}$, $b_{6}$ in the sagittal plane was as follows:

- $b_{0}$ - posterior-anterior shift of the spine from the IV

- $b_{1}$ - posterior-anterior sloping of the spine to the IV

- $b_{2}$ - overall spinal curvature (i.e., primarily curvature of thoracic kyphosis)

- $b_{3}$ and $b_{4}$ - curvature of the upper and lower parts of the spine (i.e., curvature of cervical and lumbar lordosis)

- $b_{5}$ and $b_{6}$ - residual spinal curvature of just units of millimetres

A sixth-degree polynomial was applied to describe the spinal shape in the sagittal plane since it represented the best approximation to the physiological curvature of spine with two inflexion points ${ }^{9}$. The positions of two inflexion points might be interpreted as a cervicothoracic transition and a thoracolumbar transition. These transitions split the spine into three parts: the cervical, thoracic, and lumbar spine. The curvature of the respective spinal parts could be described with angle parameters (Fig. 3) defined as follows:

- The angle of the cervical lordosis, $\alpha_{C}$, is the angle between the normal lines (perpendicular line to tangent of polynomial curve in the respective point) projected from the processus spinosus at $\mathrm{C} 3$ and the cervicothoracic transition.

- The angle of the thoracic kyphosis, $\alpha_{T}$, is the angle between the normal lines projected from the cervicothoracic transition and the thoracolumbar transition. 
Table 2. Polynomial coefficients and angle parameters in the sagittal plane in the 60 subjects in postures $\mathrm{A}, \mathrm{B}$, and $\mathrm{C}$

\begin{tabular}{|c|c|c|c|c|c|c|c|c|c|c|c|}
\hline \multirow{2}{*}{$\begin{array}{c}\text { Coefficient/ } \\
\text { angle }\end{array}$} & \multicolumn{2}{|c|}{ Posture A } & \multicolumn{2}{|c|}{ Posture B } & \multicolumn{2}{|c|}{ Posture C } & \multicolumn{3}{|c|}{ Comparison B - A } & \multicolumn{2}{|c|}{ Comparison $\mathrm{C}-\mathrm{A}$} \\
\hline & M & SD & M & SD & M & SD & $\Delta$ & $P$ & & $\Delta$ & $P$ \\
\hline $\begin{array}{ll}b_{0} & {[\mathrm{~mm}]}\end{array}$ & 7.5 & 20.0 & 21.6 & 22.8 & 37.6 & 16.7 & 14.2 & $\dagger 0.000$ & & $30.2+c$ & 0.000 \\
\hline$b_{1} \quad[\mathrm{~mm}]$ & 5.5 & 10.1 & 10.1 & 11.0 & -3.5 & 9.3 & 4.6 & $\dagger 0.000$ & & $-9.0+c$ & 0.000 \\
\hline$b_{2} \quad[\mathrm{~mm}]$ & 38.2 & 9.3 & 38.8 & 9.1 & 34.7 & 9.8 & 0.6 & 0.168 & & $-3.5+c$ & 0.002 \\
\hline$b_{3} \quad[\mathrm{~mm}]$ & 13.5 & 5 & 13.8 & 5.6 & 12.5 & 6.6 & 0.3 & 0.125 & & $-1.0+c$ & 0.060 \\
\hline$b_{4} \quad[\mathrm{~mm}]$ & -12.5 & 4.2 & -12.9 & 4.2 & -14.6 & 4.7 & -0.4 & 0.019 & & $-2.1+c$ & 0.000 \\
\hline$b_{5} \quad[\mathrm{~mm}]$ & -2.3 & 3.1 & -2.5 & 3.0 & -1.1 & 3.0 & -0.1 & 0.340 & & $1.3 \dagger c$ & 0.000 \\
\hline$b_{6} \quad[\mathrm{~mm}]$ & -3.2 & 1.9 & -2.8 & 1.6 & -1.8 & 1.8 & 0.4 & 0.003 & & $1.4 \dagger c$ & 0.000 \\
\hline$\left[{ }^{\circ}\right]$ & 30.3 & 11.8 & 29.0 & 11.5 & 25.1 & 10.1 & -1.3 & $\dagger 0.139$ & & $-5.2+c$ & 0.000 \\
\hline$\left[{ }^{\circ}\right]$ & 39.4 & 7.2 & 39.5 & 6.9 & 36.6 & 7.7 & 0.0 & 0.874 & & $-2.8+c$ & 0.001 \\
\hline$\left[{ }^{\circ}\right]$ & 39.7 & 13.7 & 38.8 & 13.8 & 42.6 & 14.3 & -0.9 & 0.053 & & $2.9+c$ & 0.001 \\
\hline
\end{tabular}

Legend

$b_{0}, b_{1}, \ldots, b_{6}$ - polynomial coefficients in the sagittal plane

$\alpha_{C}$ - angle of cervical lordosis

$\alpha_{T}$ - angle of thoracic kyphosis

$\alpha_{L}$ - angle of lumbar lordosis

$\mathrm{M}$ - arithmetic mean

SD - standard deviation

$\Delta$ - parameter difference

$\dagger-|\Delta| \geq 1 \mathrm{~mm}$

$P$ - significance of paired $t$-test

$*-P<0.05$

- The angle of lumbar lordosis, $\alpha_{L}$, is the angle between the normal lines projected from the thoracolumbar transition and the processus spinosus at L5.

\section{Statistics}

All calculations for evaluation of postural sway and spinal shape were performed using MATLAB 7.0 and the Statistics Toolbox (The MathWorks, Natick, MA). The statistical significance of changes in any parameter was assessed using the $F$-test and paired $t$-test ${ }^{14}$, with a cut-off for significance of $p=0.05$. The effect of size of parameter change was based on the absolute size of the difference. For an absolute difference of $|\Delta|<1 \mathrm{~mm}\left(1^{\circ}\right)$, the parameter change was considered as an insignificant effect of size, and vice versa.

\section{RESULTS}

\section{Influence of examination posture on postural stability}

The values of MSD for coordinates $x$ and $y$ of the individual processus spinosus in the 60 subjects in postures $\mathrm{A}, \mathrm{B}$, and $\mathrm{C}$ are given in Table 1 together with the results of the statistical tests and effect of size of differences between the postures A and B and postures A and C. The values of MSD in the coordinate $z$ did not exceed $1 \mathrm{~mm}$ and no statistical or effect size significance was found,

hence these values were not shown in the Table 1. Figure 4 shows the results of the MSDs of the processus spinosus including the mean position of the processus spinosus for all three studied postures.

The postural sway in coordinate $x$ was significantly lower for fixation posture B than for posture A, and the effect size was within the limits of significance. The reduction of postural sway in coordinate $y$ was statistically significant only for the processus spinosus between T6 and L5, and the effect size was significant only for the processus spinosus of T8 and T9. The postural sway in coordinates $x$ and $y$ was significantly lower for fixation posture $\mathrm{C}$ than for posture $\mathrm{A}$, and the effect size was significant along the entire spine except for the processus spinosus of L4 and L5 for the coordinate $x$. Taken together, fixation posture $\mathrm{C}$ was the only position that significantly increased the stability of the standing position during contact non-radiographic examination of spine.

\section{Influence of examination posture on spinal shape}

The results of the evaluation of spinal shape in the sagittal plane, including mean values, SDs of the polynomial coefficients, and angle values corresponding to the angles of cervical lordosis, thoracic kyphosis, and lumbar lordosis in postures A, B, and $\mathrm{C}$ are given in Table 2. This table also lists the effect size and the statistical significance of differences in coefficients and angles between postures A 
and $\mathrm{B}$ and postures $\mathrm{A}$ and $\mathrm{C}$. The paired $t$-test was used for statistical comparisons.

Effect size and statistically significant differences in polynomial coefficients $b_{0}$ and $b_{1}$ were found between postures $\mathrm{A}$ and $\mathrm{B}$, whereas differences in coefficients $b_{4}$ and $b_{6}$ were statistically significant but there was no significant effect size. The difference in the angles of cervical lordosis achieved significant effect size, but this was not statistically significant. It might be concluded that the spinal shift from ideal vertical and spinal slope was higher for fixation posture $\mathrm{B}$ than for posture $\mathrm{A}$, but the curvature of the individual sectors was the same. The results of the spinal shape analysis in the sagittal plane are shown in Fig. 3.

Effect size and statistically significant differences were found between postures $\mathrm{A}$ and $\mathrm{C}$ in terms of all polynomial coefficients and all angle polynomial parameters except for coefficient $b_{3}$ which was not statistically significant. Compared to posture $\mathrm{A}$, fixation posture $\mathrm{C}$ exhibits an increased deviation of the spinal shape from ideal vertical, a decreased spinal slope, a flattened cervical lordosis and thoracic kyphosis, and an increased lumbar lordosis curvature.

\section{DISCUSSION}

Accurate and timely diagnosis of type and degree of a spinal deformity is basic to correct treatment, either surgical or conservative ${ }^{15,16}$. The most common examination method is a radiological evaluation which involves measurement of rotation and torsion of the vertebrae ${ }^{17}$. Long-term follow-ups are required in the majority of cases of spinal deformity leading to increased cumulative radiation dose. Another limitation of the radiographic method is that it cannot make X-rays in the frontal and sagittal plane at the same time unless computed tomography is used which enables three-dimensional reconstruction ${ }^{18}$. However, computed tomography exposes the patient to a much higher level of radiation than that for simple radiography. Alternative, non-radiographic methods are therefore being researched. In addition to eliminating the disadvantages of radiological examination, these techniques should also use modern technologies with their increased reliability and accuracy, and that allow dynamic examinations ${ }^{6,9}$.

In theory, these methods are based on the spinal shape being sufficiently represented by projections of anatomic points on the body surface ${ }^{19}$. The coordinates of these points are measured from the surface of the back using mechanical, optical, or ultrasound sensors. Subsequent computer processing enables both the $3 \mathrm{D}$ reconstruction of spinal shape and the calculation of other clinically important parameters ${ }^{20}$. On the other hand, all of these methods depend on stable posture during the examination period. In this regard, postural sway, breathing and other sources of postural instability can be a problem.

The results of this study show that the examination position used for spine diagnostics can significantly in- fluence the spinal shape and degree of postural sway. Theoretically, precise and reliable diagnostics of spinal shape require an examination position that minimizes both the postural sway and changes in spinal shape. The examination positions evaluated in our study do not simultaneously fulfil both of these requirements. Postures $\mathrm{A}$ and $\mathrm{B}$ do not address the requirement of sufficient postural stability, and although posture $\mathrm{C}$ significantly reduces the degree of postural sway, it does not address the requirement of a minimal influence on spinal shape.

Selecting the most appropriate examination position for spinal shape diagnostics does not depend on the examination method, and hence is applicable to both non-invasive, surface examinations and radiological examinations. The influence of various modifications of examination positions applicable to the X-ray method in the frontal plane has been described previously ${ }^{21}$, but that study did not consider postural sway. Various examination positions were used in the various methods of surface diagnostics of spinal shape, such as the Arcometer ${ }^{1}$, the BACES system $^{2}$, the Metrecom system ${ }^{22}$, the Ortelius $800^{\mathrm{TM}}$ system $^{6}$, Posturometr-S ${ }^{23}$, and the SpinalMouse system ${ }^{24}$. These variations preclude direct comparisons of the results of such studies. For the BACES system, the Metrecom system and Posturometr-S, the use of special fixation devices to increase postural stability was proposed but the degree of postural sway was not quantified and the influence of the fixation procedure on the spinal shape was not discussed. Other reports on surface spinal shape diagnostics using X-ray ${ }^{1,4}$ have not addressed the influence of various examination positions. Overall, close attention should be paid to standardizing the measurement protocol including the definition of examination posture.

\section{CONCLUSIONS}

We found that postural sway could strongly influence on the variability of method for spinal shape examination. Therefore, further research is necessary to find out such examination position that would increase the reproducibility and validity of spinal examinations. In addition, this study has supported further development of non-radiographic computer-assisted assessment of spinal shape because they are three-dimensional, quick, and reliable.

\section{ACKNOWLEDGMENT}

This study was supported by a research grant from the Ministry of Education, Youth, and Sports of the Czech Republic (No. MSM 6198959221) entitled "Physical Activity and Inactivity of the Inhabitants of the Czech Republic in the Context of Behavioral Changes." 


\section{ETHICS APPROVAL}

This study was approved by the Ethical Committee of the Faculty of Physical Culture of Palacky University in Olomouc. All the subjects participated in this study were volunteers, and had given their informed consent.

\section{REFERENCES}

1. D'Osualdo F, Schierano S, Iannis M. Validation of clinical measurement of kyphosis with a simple instrument, the arcometer. Spine 1997; 22:408-13.

2. D’Osualdo F, Schierano S, Soldano FM, Isola M. New tridimensional approach to the evaluation of the spine through surface measurement: the BACES system. J Med Eng Technol 2002; 26:95105.

3. Kolisko P, Salinger J, Novotny J, Vychodil R. Diagnostic of spine disorders by positional tracking device. Acta Universitatis Palackianae Olomucensis Gymnica 1996; 26:25-30.

4. Leroux MA, Zabjek K, Simard G, Badeaux J, Coillard C, Rivard $\mathrm{CH}$. A noninvasive anthropometric technique for measuring kyphosis and lordosis: an application for idiopathic scoliosis. Spine 2000; 25:1689-94.

5. Mannion AF, Knecht K, Balaban G, Dvorak J, Grob D. A new skin-surface device for measuring the curvature and global and segmental ranges of motion of the spine: reliability of measurements and comparison with data reviewed from the literature. Eur Spine J 2004; 13:122-36.

6. Ovadia D, Bar-On E, Fragniere B, Rigo M, Dickman D, Leitner J, et al. Radiation-free quantitative assessment of scoliosis: a multi center prospective study. Eur Spine J 2007; 16:97-105.

7. Chen PQ, Wang JL, Tsuang YH, Liao TL, Huang PI, Hang YS. The postural stability control and gait pattern of idiopathic scoliosis adolescents. Clin Biomech (Bristol, Avon) 1998; 13(Suppl 1):S52-S58.

8. Nault ML, Allard P, Hinse S, Le Blanc R, Caron O, Labelle H, et al. Relations between standing stability and body posture parameters in adolescent idiopathic scoliosis. Spine 2002; 27:1911-7.

9. Krejci J. System for diagnosing spinal shape in humans. Palacky University Olomouc. Czech Republic. 2007. Available from:
www.upol.cz//fileadmin/user_upload/FTK-katedry/biomechanika/ krejci_doctoral_thesis.pdf. Accessed January 17, 2008.

10. European Co-operation in Accreditation. EA-4/02 Expression of the uncertainty of measurement in calibration. 1999.

11. Kubackova L. Foundations of experimental data analysis. Boca Raton-Ann Arbor-London-Tokyo: CRC Press; 1992.

12. Riegerova J, Szotkowska J, Krejci J. Diagnostics of posture and spine shape using the DTP-2 position detector for U3A senior students before and after an intervention procedure by a targeted excercise program. Auxologija a promocja zdrowia 2007; 4:25364.

13. Rektorys K. Survey of applicable mathematics. Cambridge, MA: MIT Press; 1969.

14. Dowdy S, Wearden S. Statistics for research. New York: John Wiley \& Sons; 1983.

15. Weinstein SL, Dolan LA, Cheng JC, Danielsson A, Morcuende JA. Adolescent idiopathic scoliosis. Lancet 2008; 371(9623):152737.

16. Goldberg CJ, Moore DP, Fogarty EE, Dowling FE. Scoliosis: a review. Pediatr Surg Int 2008; 24:129-44

17. Davies A, Saifuddin A. Imaging of painful scoliosis. Skeletal Radiol. Epub ahead of print, July 12, 2008.

18. Kotwicki T. Evaluation of scoliosis today: examination, X-rays and beyond. Disabil Rehabil 2008; 30:742-51.

19. Drerup B, Hierholzer E. Automatic localization of anatomical landmarks on the back surface and construction of a body-fixed coordinate system. J Biomech 1987; 20:961-70.

20. Huysmans T, Haex B, Van Audekercke R, Vander Sloten J, Van der Perre G. Three-dimensional mathematical reconstruction of the spinal shape, based on active contours. J Biomech 2004; 37:17938

21. Marks MC, Stanford CF, Mahar AT, Newton PO. Standing lateral radiographic positioning does not represent customary standing balance. Spine 2003; 28:1176-82.

22. Norton BJ, Hensler K, Zou D. Comparisons among noninvasive methods for measuring lumbar curvature in standing. J Orthop Sports Phys Ther 2002; 32:405-14.

23. Sliwa W, Sliwa K. Wady postawy ciala i ich ocena. POSMED; Wroclaw, Poland; 2001.

24. Carlucci L, Chiu JC, Cilifford TJ. Spinal Mouse for assessment of spinal mobility. J Minim Invasive Spinal Tech 2001; 1:30-31. 
